\title{
Comparison of Ganglion Cell Complex Defect Patterns of Exfoliative Glaucoma and Primary Open Angle Glaucoma using Optıcal Coherence Tomography
}

\author{
Damla Aydın ${ }^{1 *}$, Tuncay Kusbecı ${ }^{2}$, Feyza Gedız ${ }^{2}$ and Bora Yuksel ${ }^{2}$ \\ ${ }^{1}$ Denizli Civril State Hospital, Department of Ophthalmology, Turkey \\ ${ }^{2}$ Bozyaka Training and Research Hospital, Department of Ophthalmology, Turkey
}

Submission: November 27, 2017; Published: December 18, 2017

*Corresponding author: Damla Aydın, Denizli Civril State Hospital, Department of Ophthalmology, 20650, Civril/DENIZLI, Tel: +90-0534-825-2122; +90-258-713-10-22; Fax: +90-258-713-21-00; Email: damlaydinn@hotmail.com

\begin{abstract}
Purpose: To compare GCC defect patterns and ganglion cell complex (GCC) thicknesses in exfoliative glaucoma (XFG) and primary open angle glaucoma (POAG) by using optical coherence tomography (OCT) and evaluate if exfoliation material accumulation plays an independent role in optic nerve damage.

Methods: 32 XFG and 32 POAG patients were enrolled the study. RNFL and GCC measurements are performed by Cirrus HD-OCT 4000 (Carl Zeiss Meditec, Dublin, CA, USA). GCC deviation map was used to determine GCC defect patterns.

Results: Mean and minimum GCC thicknesses were significantly thinner in XFG than POAG ( $p=0.038, p=0.025$, respectively). GCC thicknesses in all quadrants except in superotemporal quadrant were significantly thinner in XFG than POAG ( $<<0.05$ for each quadrant except superotemporal quadrant, $\mathrm{p}=0.101$ for superotemporal quadrant). There was no significant difference according to GCC defect patterns between two groups ( $p=0.187$ ). But GCC defect patterns were significantly different between two groups ( $p=0.023$ ) in advanced glaucoma, diffuse severe type GCC defect was the most frequently observed in XFG and more frequent in XFG than POAG.

Conclusion: XFG is related with thinner RNFL and GCC when compared with POAG although the smilar visual field test results. In advanced glaucoma diffuse severe type GCC defect was observed more frequently in XFG than POAG. As the severity of glaucoma increased, GCC defect patterns tended to be more severe in XGF than POAG.
\end{abstract}

Keywords: Exfoliative glaucoma; Ganglion cell defect pattern; Optical coherence tomography; Primary open angle glaucoma

\section{Introduction}

FaGlaucoma is the second most common cause of blindness in the world [1]. Open angle glaucomas (OAG) comprise the majority of the cases [2,3]. OAG is defined as a progressive damage of retinal ganglion cells and their respective axons resulting neuroretinal rim and visual field loss with open iridocorneal angle [4]. Whereas primary open angle glaucoma (POAG) is the most common form of OAG, exfoliative glaucoma $(\mathrm{XFG})$ is the most identifiable cause of open angle glaucoma $[5,6]$.

POAG is without an identifiable cause of aqueous outflow resistance, but in XFG exfoliation material accumulation leads aqueous outflow resistance [7]. While not all people with exfoliation material accumulation develop glaucoma, those with XFG tend to have a higher IOP at diagnosis than those with POAG [8]. In addition diurnal IOP fluctuation is higher in XFG than POAG [9]. Also, it was thought that exfoliation material accumulation may predispose to glaucomatous optic nerve damage without IOP elevation and it has been considered as an independent risk factor for optic nerve damage [10-12].

Optical coherence tomography (OCT) can provide quantitative analysis of retinal nerve fiber layer and ganglion cell-inner plexiform cell layer. Standart automatized perimetry (SAP) shows functional changes due to ganglion cell loss in glaucoma cases, but OCT assesses directly ganglion cells and their axons and can detect ganglion cell loss earlier than SAP $[13,14]$. Evaluation of GCC defect patterns of XFG and POAG patients by using OCT can help us to understand the difference between the optic nerve damage mechanisms of XFG and POAG.

In our study, we evaluated retinal nerve fiber layer (RNFL) thickness, retinal ganglion cell complex (GCC) thickness and GCC defect patterns of XFG and POAG. We compared the results to 
show if there is structural difference between XFG and POAG despite the smilar SAP results to investigate if exfoliation itself causes optic nerve damage.

\section{Methods}

32 POAG and 32 XFG patients were enrolled the study and investigated prospectivelyin the Ophthalomolgy Department of Izmir Bozyaka Training and Research Hospital. The study was conducted in accordance with the Declaration of Helsinki. The study was approved by the Medical Ethical Committee. Informed consent was obtained from all patients.

Detailed ophthalmological examination including best corrected visual acuity (BCVA), intraocular pressure (IOP) measurement by Goldmann applanation tonometry, slit lamp biomicroscopy, gonioscopy by Goldmann three mirror lens and dilated fundus examination by $90 \mathrm{D}$ lens were performed on all cases. Optic disc changes like cup to disc ratio $>0.7$, inter-eye cup asymmetry $>0.2$, neuroretinal rim notches, focal thinning, disc hemorrhage and vertical elongation of optic cup were evaluated as glaucomatous optic disc.

SAP was performed on all cases twice with Humphrey Visual Field Analyzer (Carl Zeiss Meditec, Dublin, Ireland) by 30-2 Swedish Interactive Threshold Algorithmrhytm (SITA) standard program. Only results with fixation loss $\leq 20 \%$, false negative and false positive ratio $\leq 15 \%$ in both tests were accepted as reliable. Mean deviation (MD) and pattern standard deviation (PSD) values were recorded. Glaucoma hemifield test (GHT) outside normal limits, abnormal pattern standard deviation with $\mathrm{p}$ value $<5 \%$ and a cluster of three or more points in the pattern deviation plot in a single hemifield with $\mathrm{p}$ values $<5 \%$, one of which must have a p value $<1 \%$ were evaluated as glaucomatous visual field defect. Glaucoma severity was classified as mild, moderate and advanced to the modified Hodapp-AndersonParrish grading scale based on the MD results of SAP. Mild, moderate and advanced were defined as $\mathrm{MD} \geq-6 \mathrm{~dB}$, MD between -6 and $12 \mathrm{~dB}, \mathrm{MD} \leq-12 \mathrm{~dB}$, respectively.

The presence of any ocular disease such as vitreoretinal pathologies, ocular inflammation, optic neuropathies, previous ocular surgery and laser treatment, ocular trauma history, refractive errors exceeding 5 diopters spheric and 3 diopters cylindric, optic media opacities such as corneal opacities and cataract (Grade 1- 3 nucleus opacification, nucleus color and cortical changes according to Lens Opacities Classification System III) were exclusion criterias. Systemic and neurologic diseases such as diabetes mellitus, hypertension, Alzheimer disease and vascular dementia were also excluded.

POAG was defined as open angle in gonioscopy, glaucomatous optic disc changes and glaucomatous visual field defect without an identifiable cause of optic nerve damage. XFG was defined as presence of detectable exfoliation material by slit lamp examination at the pupillary border or on the anterior lens capsule after pupillary dilatation, open angle in gonioscopy, glaucomatous optic nerve damage and glaucomatous visual field defect.

OCT measurements were performed using Cirrus HDOCT 4000 (Carl Zeiss Meditec, Dublin, CA, USA) after pupillary dilatation. Images with visible eye motion, blinking artefacts and those with signal strength $<6$ were excluded. At optic disc cube protocol, $6 \times 6 \mathrm{~mm}$ area was scanned with $200 \times 200$ axial scan (A-scan). Average cup/disc (C/D) ratio, vertical C/D ratio were calculated. Retinal nerve fiber layer (RNFL) thickness were measured by scanning three consecutive 360 degree circular scans with a diameter of $3.4 \mathrm{~mm}$ centered on the optic disc. Mean RNFL thickness, average RNFL thickness in four quadrants (superior, nasal, inferior and temporal) were calculated automatically. GCC thickness were calculated by measuring the thickness between RNFL-Ganglion Cell (GC) boundary and Inner Plexiform Layer(IPL)-Inner Nuclear boundary in an elliptical annulus area centered on the fovea. (vertical inner and outer radii of 0.5 and $2.0 \mathrm{~mm}$; horizontal inner and outer radii of 0.6 and $2.4 \mathrm{~mm}$, respectively) GCC (GC+IPL) thicknesses were calculated in six quadrants (superior, superonasal, inferonasal, inferior, inferotemporal and superotemporal). Mean and minimum GCC thicknesses were calculated. Minimum GCC thickness means the average GCC thickness from the radius with the lowest average among the 360 radiuses.

GCC deviation map was used to determine GCC defect patterns. Abnormal GCC thickness in the deviation map is shown by yellow and red at the $\% 5$ and $\% 1$ levels, respectively. The abnormal area deliniated manually and its area measured using the Image J programme (version 1.47). Areas were summed when there were multiple abnormal areas. Measured values were converted to the values fit to the scan area of the deviation map which was a elliptical annulus area on the $6 \times 6 \mathrm{~mm} 2$ square area of the macular scan. GCC defect patterns were classified as minimal, inner, outer, diffuse mild, diffuse severe, inferior confined, inferior dominant, superior confined and superior dominant. The inner defect was defined as circumferential GCC defect within vertical inner and outer radii of 0.5 and $1 \mathrm{~mm}$ and within horizontal inner and outer radii of 0.6 and $1.2 \mathrm{~mm}$ from the fovea, respectively. The outer defect was defined as a circumferential GCC defect outside a vertical radius of $1 \mathrm{~mm}$ and horizontal radius of $1.2 \mathrm{~mm}$ from the fovea. The diffuse mild defect was defined as a general yellow or red pattern in less than half of the scan area without hemifields preponderance. The diffuse severe defect was defined as as yellow or red colour pattern seen in more than half size of the scan area without hemifield preponderance. The inferior confined and superior confined defects were defined as yellow or red colour patterns in only the corresponding hemifield. The inferior dominant and superior dominant defects were defined as yellow or red patterns exceeding horizontal midline that were dominant in the corresponding hemifield [15]. GCC defect patterns were shown in Figure 1. 


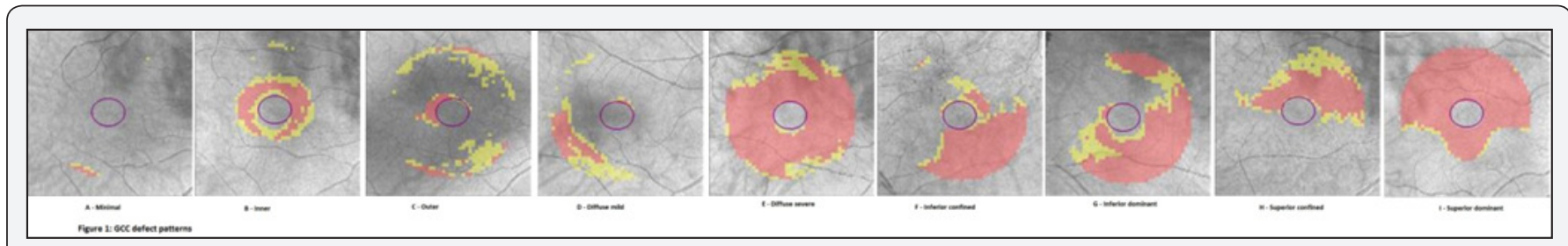

Figure 1: GCC defect patterns.

Statistical analysis was performed using SPSS 20.0. The chisquare test was used to compare categorical data. Unpaired t test was used to compare RNFL and GCC thicknesses between two groups. The chi-square test was used to compare GCC patterns between two groups. A p value less than 0.05 was accepted as statistically significant.

\section{Results}

Table 1 shows the demographic and clinical characteristics of groups. In XFG the mean IOP was $2.54 \mathrm{~mm} \mathrm{Hg}$ higher than in POAG, but the difference was not statistically significant $(p=0.075)$. There were no significant difference between POAG and XFG according to BCVA, MD value, PSD value, average cup/disc ratio, and vertical cup/disc ratio $(p>0.05$ for each parameter). There was no significant difference between two groups according to the distribution of the severity of glaucoma $(\mathrm{p}=0.301)$.

RNFL parameters were summarized in Table 2. Mean RNFL thickness was thinner in XFG than POAG ( $p=0.042)$. Also, RNFL was thinner in superior and inferior quadrants in XFG than POAG ( $p=0.043, p=0.047$, respectively). GCC parameters were summarized in Table 3. Mean and minimum GCC thicknesses were significantly thinner in XFG than POAG $(p=0.038, p=0.025$, respectively). GCC thicknesses in all quadrants except in superotemporal quadrant were significantly thinner in XFG than POAG $(\mathrm{p}<0.05$ for each quadrant except superotemporal quadrant, $\mathrm{p}=0.101$ for superotemporal quadrant).

Table 1: Demographic and clinical characteristics of XFG and POAG.

\begin{tabular}{|c|c|c|c|}
\hline & XFG (n=32) & POAG (n=32) & P* \\
\hline Age (year) & $67.01 \pm 8.37$ & $67.51 \pm 9.33$ & $0.818^{*}$ \\
\hline Sex & $12 \mathrm{~F}$ & $16 \mathrm{~F}$ & $0.450^{* *}$ \\
\hline BCVA (logMAR) & $20 \mathrm{M}$ & $16 \mathrm{M}$ & $0.065^{*}$ \\
\hline IOP (mm-Hg) & $0.37 \pm 0.43$ & $0.21 \pm 0.16$ & $0.075^{*}$ \\
\hline C/D average & $18.53 \pm 4.51$ & $16.62 \pm 3.88$ & $0.425^{*}$ \\
\hline C/D vertical & $0.71 \pm 0.13$ & $0.68 \pm 0.17$ & $0.510^{*}$ \\
\hline MD & $0.70 \pm 0.14$ & $-8.18 \pm 7.31$ & $0.129^{*}$ \\
\hline PSD & $-11.08 \pm 6.77$ & $4.42 \pm 3.16$ & $8(25.0 \%)$ \\
Severity & $5.78 \pm 2.95$ & $15(46.8 \%)$ & $0.103^{*}$ \\
\hline Mild & $6(18.8 \%)$ & $9(28.2 \%)$ & $0.301^{* *}$ \\
\hline
\end{tabular}

*independent $\mathrm{t}$ test,${ }^{* *} \mathrm{chi}$-square test

Data are presented as mean \pm standard deviation.

XFG: Exfoliative Glaucoma; POAG: Primary Open Angle Glaucoma; BCVA: Best Corrected Visual Acuity; IOP: Intraocular Pressure; C/D: Cup/Disk ratio; MD: Mean Deviation; PSD: Pattern Standard Deviation

Table 2: RNFL parameters of XFG and POAG.

\begin{tabular}{|c|c|c|c|}
\hline & XFG $(\mathbf{n}=\mathbf{3 2})$ & POAG (n=32) & P* \\
\hline RNFL average & $65.53 \pm 14.74$ & $72.97 \pm 13.58$ & 0.042 \\
\hline RNFL superior & $73.87 \pm 22.25$ & $84.69 \pm 19.61$ & 0.043 \\
\hline RNFL nasal & $57.56 \pm 13.22$ & $60.78 \pm 11.07$ & 0.295 \\
\hline RNFL inferior & $78.72 \pm 24.30$ & $90.44 \pm 21.89$ & 0.047 \\
\hline RNFL temporal & $51.91 \pm 13.26$ & $57.03 \pm 11.63$ & 0.105 \\
\hline
\end{tabular}


Data are presented as mean \pm standard deviation

$\mathrm{p}<0.05$ shown in boldface, *independent $\mathrm{t}$ test

RNFL: Retinal Nerve Fiber Layer; XFG: Exfoliative Glaucoma; POAG: Primary Open Angle Glaucoma

Table 3: GCC parameters of XFG and POAG.

\begin{tabular}{|c|l|l|l|}
\hline & XFG (n=32) & POAG (n=32) & $69.41 \pm 10.66$ \\
\hline GCC average & $63.19 \pm 12.76$ & $61.72 \pm 13.67$ & 0.038 \\
\hline GCC minimum & $53.53 \pm 14.87$ & $68.31 \pm 11.71$ & $71.44 \pm 11.49$ \\
\hline GCC superior & $61.12 \pm 12.34$ & $71.40 \pm 10.82$ & 0.020 \\
\hline GCC superonasal & $62.87 \pm 13.62$ & $68.97 \pm 11.26$ & 0.010 \\
\hline GCC inferonasal & $62.37 \pm 16.00$ & $68.94 \pm 13.72$ \\
\hline GCC inferior & $60.18 \pm 15.69$ & $65.97 \pm 11.40$ \\
\hline
\end{tabular}

Data are presented as mean \pm standard deviation

$p<0.05$ shown in boldface, *independent $t$ test

GCC: Ganglion Cell Complex; XFG: Exfoliative Glaucoma; POAG: Primary Open Angle Glaucoma

GCC defect patterns were summarized in Table 4. There was no significant difference according to GCC defect patterns between two groups $(\mathrm{p}=0.187)$. There was no significant difference between mild XFG and POAG; moderate XFG and POAG according to GCC defect patterns $(p=0.380, p=0.785$, respectively). In advanced glaucoma, GCC defect patterns were significantly different between two groups $(\mathrm{p}=0.023)$, diffuse severe type GCC defect was the most frequently observed defect pattern in advanced XFG and more frequent in advanced XFG than advanced POAG. In advanced POAG diffuse mild and diffuse severe type defects were the most frequent patterns.

Table 4: GCC defect patterns of XFG and POAG.

\begin{tabular}{|c|c|c|c|}
\hline & $\mathrm{XFG}(\mathrm{n}=32)$ & POAG $(n=32)$ & $\mathbf{P}^{*}$ \\
\hline Minimal & $3(9.3 \%)$ & $5(15.6 \%)$ & \multirow{9}{*}{0.187} \\
\hline Inner & $4(12.5 \%)$ & $2(6.0 \%)$ & \\
\hline Outer & $0(0.0 \%)$ & $1(3.0 \%)$ & \\
\hline Diffuse mild & $7(21.8 \%)$ & $8(25.0 \%)$ & \\
\hline Diffuse severe & $8(25.0) \%$ & $5(15.6 \%)$ & \\
\hline Inferior confined & $3(9.3 \%)$ & $1(3.0 \%)$ & \\
\hline Inferior dominant & $4(12.5 \%)$ & $4(12.5 \%)$ & \\
\hline Superior confined & $1(3.0 \%)$ & $2(6.0 \%)$ & \\
\hline Superior dominant & $2(6.0 \%)$ & $4(12.5 \%)$ & \\
\hline
\end{tabular}

*independent $t$ test, XFG: Exfoliative Glaucoma, POAG: Primary Open Angle Glaucoma

\section{Discussion}

Mechanism of the ganglion cell loss in glaucoma has not been totally understood yet but some theories have been suggested. The level of IOP is related to ganglion cell death. IOP-induced stress causes compression, deformation and remodeling of lamina cribrosa with consequent mechanical axonal stress and disruption of axonal transport [16]. Glaucomatous optic neuropathy can occur also in normal IOP levels, due to low cerebrospinal fluid pressure in the optic nerve subarachnoid space resulting in a large pressure gradient across the lamina cribrosa [17]. Impaired microcirculation, altered immunity, excitotoxicity and oxidative stress are the other causes of glaucomatous optic nerve damage.
Despite the common mechanisms of glaucomatous optic neuropathy in POAG and XFG, it has been suggested that exfoliation material accumulation may predispose to glaucomatous optic nerve damage without IOP elevation and it has been considered as an independent risk factor for optic nerve damage [10-12].

Changes in exon 1 of the lysyl oxydase like protein 1 gene (LOXL1) on chromosome 15q24.1 that catalyzes the formation of elastin fibers found to be a major component of the exfoliation material results exfoliation material accumulation and increase the risk for XFG [18]. LOXL1 expression is detected in ocular tissues such as lamina cribrosa, lens epithelium, cornea, ciliary muscle and trabecular meshwork $[19,20]$. It has been suggested 
that exfoliation material accumulation in some ocular tissues such as short posterior ciliary arteries, vortex veins, central retinal vein, optic nerve sheath and lamina cribrosa may cause optic nerve damage [21,22]. Netland et al. [23] showed elastosis in lamina cribrosa in exfoliative eyes that may cause predisposition of optic nerve damage [23]. It has been suggested that exfoliation syndrome is related with decreased retrobulber blood flow, so impaired blood flow may be responsible of optic nerve damage [24]. But in a recent study no retrobulber blood flow difference has been found between exfoliative eyes and healthy eyes [25]. Decreased microvascular blood flow in optic nerve head and peripapillary retina has been showed by Heidelberg retina flowmeter in XFS [26].

OCT provides a quantitative analysis of optic nerve head, RNFL and GCC; also prevents interobserver variability for evaluation of optic disk by slit lamp biomicroscopy or optic disk photography that are subjective methods. Furthermore OCT assesses structural changes in retina directly as SAP visualizes functional changes due to ganglion cell loss. So, OCT detects optic nerve damage earlier than SAP $[27,28]$. Advent of automatic segmentation of macular layers by new algorithms has provided ganglion cell analysis that discards the disadvantage of measuring the outer retinal layers not effected in glaucoma. Analysing macular ganglion cells provides a less variable analysis than RNFL analysis among normal individuals [29]. Some studies have showed that GCC and RNFL parameters have similar performance to assess any stage of glaucoma [30,31]. But in other studies it has been suggested that although GCC parameters have similar performance to RNFL parameters, RNFL parameters are slightly better than GCC parameters [32,33]. So, it has been suggested that GCC and RNFL parameters together are more sensitive than alone $[34,35]$. In our study, we evaluated RNFL and GCC thicknesses, also GCC defect patterns of XFG and POAG patients; compared the results.

Sorkhabi et al. [36] found that mean RNFL thickness was thinner in XFS than healthy subjects [36]. Yuksel et al. [37] reported that in unilateral XFS, RNFL was significantly thinner in all quadrants except nasal quadrant in the exfoliative eye than healthy eye [37]. Barkana et al. [38] reported that in unilateral hypertensive exfoliation syndrome RNFL was thinner in the hypertensive eyes [38]. In our study mean RNFL thickness, RNFL thickness in superior and inferior quadrants were thinner in XFG than POAG.

In our study, mean and minimum GCC thicknesses were significantly thinner in XFG than POAG. Also, GCC thicknesses in all quadrants except in superotemporal quadrant were significantly thinner in XFG than POAG.

In this study GCC defect patterns were classified as minimal, inner, outer, diffuse mild, diffuse severe, inferior confined, inferior dominant, superior confined and superior dominant. Tan et al. [32] reported that macular inner nuclear layer loss was more significant in inferior perifoveal region in perimetric and preperimetric glaucoma patients [32]. Kotera et al. [39] reported that inner retinal layers were thinner especially in inferior quadrant than superior quadrant and in temporal quadrant than nasal quadrant in glaucoma suspects and preperimetric glaucoma patients than healthy individuals [39]. Hood et al. [40] reported that ganglion cell plus inner plexiform layers were thinner in the inferior region in glaucoma patients [40]. Jeong et al. [15] showed that in mild and moderate stage POAG patients the inferior confined type ganglion cell patterns was more frequent, in advanced stage POAG patients diffuse severe type ganglion cell pattern was more frequent [15]. We found that GCC defect patterns were not significantly different between XFG and POAG. The most frequent GCC defect pattern was the diffuse severe type in XFG and diffuse mild in POAG. Diffuse type GCC defects were more frequently seen in both groups than localized defects like inferior confined, inferior dominant, superior confined and superior dominant. But in advanced glaucoma GCC defect patterns were significantly different between XFG and POAG, diffuse severe type GCC pattern defect was the most frequently observed in XFG and more frequent in XFG than POAG. As the severity of glaucoma increased, GCC pattern defect tended to be more severe in XFG than POAG.

Best of our knowledge there was no study in literature that compares RNFL and GCC parameters of XFG and POAG. We found that in XFG, RNFL and GCC were thinner than POAG as MD and PSD values were similar; but there was no difference in GCC defect patterns except advanced glaucoma. XFG is an agressive glaucoma type that is characterized by higher IOP at diagnosis, more diurnal fluctation of IOP, poor response to medical treatment when compared with POAG. In our study, the mean IOP was $2.54 \mathrm{~mm}-\mathrm{Hg}$ higher in XFG than POAG. But we haven't performed diurnal IOP measurements, so higher diurnal fluctuation in XFG patients might occur. So, higher IOP or diurnal fluctation that we could not rule out may cause thinner RNFL and GCC results. The limitations of our study are no diurnal IOP measurements were performed and limited patient number.

\section{Conclusion}

In conclusion, XFG is related with thinner RNFL and GCC when compared with POAG although the smilar SAP results. In advanced glaucoma diffuse severe type GCC defects was observed more frequently in XFG than POAG. It can be said that the axonal damage is higher in XFG than POAG despite the smilar SAP results and stages. Higher IOP and diurnal fluctuation can cause more optic nerve damage in XFG, also exfoliation material itself can cause additional damage but further studies are needed if exfoliation material accumulation is an independent risk factor for optic nerve damage.

\section{References}

1. Resnikoff S, Pascolini D, Etya'ale D, Kocur I, Pararajasegaram R, et al. (2004) Global data on visual impairment in the year 2002. Bull World Health Organ 82(11): 844-851. 
2. Friedman DS, Wolfs RC, O'Colmain BJ, Klein BE, Taylor HR, et al. (2004) Eye Diseases Prevalence Research Group. Prevalence of open-angle glaucoma among adults in the United States. Arch Ophthalmol 122(4) 532-538.

3. Day AC, Baio G, Gazzard G, Bunce C, Azuara-Blanco A, et al. (2012) The prevalence of primary angle closure glaucoma in European derived populations : a systematic review. Br J Ophthalmol 96(9): 1162-1167.

4. Foster PJ, Buhrmann R, Quigley HA, Johnson GJ (2002) The definition and classification of glaucoma in prevalence surveys. Br J Ophthalmol 86(2): 238-242.

5. Weinreb RN, Khaw PT (2004) Primary open angle glaucoma. Lancet 363(9422): 1711-1720.

6. Schlotzer-Schrehardt U, Naumann GO (2006) Ocular and systemic pseudoexfoliaon syndrome. Am J Ophthalmol 141(5): 921-937.

7. Gharagozloo NZ, Baker RH, Brubaker RF (1992) Aqueous Dynamics in exfoliation syndrome. Am J Ophthalmol 114(4): 473-478.

8. Teus MA, Castejón MA, Calvo MA, Pérez-Salaíces P, Marcos A (1998) Intraocular pressure as a risk factor for visual field loss in pseudoexfoliative and in primary open-angle glaucoma. Ophthalmology 105(12): 2225-2229.

9. Altintaş O, Yüksel N, Karabaş VL, Qağlar Y (2004) Diurnal intraocular variation in pseudoexfoliation syndrome. Eur J Ophthalmol 14(6): 495-500.

10. Ekström C (1993) Elevated intraocular pressure and pseudoexfoliation of the lens capsule as risk factor for chronic open angle glaucoma. A population based five year follow-up study. Acta Ophthalmol (Copenh) 71(2): 189-195.

11. Mitchell P, Wang JJ, Hourihan F (1999) The relationship between glaucoma and pseudoexfoliation. The Blue Mountains Eye Study. Arch Ophthalmol 117(110): 1319-1324.

12. Davanger M, Ringvold A, Blika S (1991) Pseudo-exfoliation, IOP, and glaucoma. Acta Ophthalmol (Copenh) 69(5): 569-573.

13. Medeiros FA, Alencar LM, Zangwill LM, Bowd C, Sample PA, et al. (2009) Prediction of functional loss in glaucoma from progressive optic disc damage. Arch Ophthalmol 127(10): 1250-1256.

14. Sommer A, Katz J, Quigley HA, Miller NR, Robin AL, et al. (1991) Clinically detectable nerve fiber atrophy precedes the onset of glaucomatous field loss. Arch Ophthalmol 109(1): 77-83.

15. Jeong JS, Kang MG, Kim CY, Kim NR (2015) Pattern of Macular Ganglion Cell-Inner Plexiform Layer Defect Generated by Spectral-Domain OCT in Glaucoma Patients and Normal Subjects. J Glaucoma 24(8): 583-590.

16. Burgoyne CF, Downs JC, Bellezza AJ, Suh JK, Hart RT (2005) The optic nerve head as a biomechanical structure: a new paradigm for understanding the role of IOP-related stres and strain in the pathophysiology of glaucomatous optic nerve head damage. Prog Retin Eye Res 24(1): 39-73.

17. Wang N, Xie X, Yang D, Xian J, Li Y, et al. (2012) Orbital cerebrospinal fluid space in glaucoma: the Beijing Intracranial and Intraocular Pressure (iCOP) study. Ophthalmology 119(10): 2065-2073.

18. Thorleifsson G, Magnusson KP, Sulem P, Walters GB, Gudbjartsson DF, et al. (2007) Common sequence variants in the LOXL1 gene confer suspectibility to exfoliation glaucoma. Science 317(5843): 1397-1400.

19. Schlötzer-Schrehardt U, Pasutto F, Sommer P, Hornstra I, Kruse FE, et al. (2008) Genotype-correlated expression of lysyl oxidase-like 1 in ocular tissues of patients with pseudoexfoliation syndrome/glaucoma and normal patients. Am J Pat 173(6): 1724-1735.

20. Khan TT, Li G, Navarro ID, Kastury RD, Zeil CJ, et al. (2010) LOXL1 expression in lens capsule tissue specimens from individuals with pseudoexfolaition syndrome and glaucoma. Mol Vis 16: 2236-2241.
21. Schlötzer-Schrehardt U, Küchle M, Naumann GOH (1991) Electronmicroscopic identification of pseudoexfoliation material in extrabulbar tissue. Arch Ophthalmol 109(4): 565-570.

22. Gottanka J, Flügel-Koch C, Martus P, Johnson DH, Lütjen-Drecoll E (1997) Correlation of pseudoexfoliative material and optic nerve damage in pseudoexfoliation syndrome. Invest Ophthalmol Vis Sci 38(12): 2435-2446.

23. Netland PA, Ye H, Streeten BW, Hernandez MR (1995) Elastosis of the lamina cribrosa in pseudoexfoliation syndrome with glaucoma. Ophthalmology 102(6): 878-886.

24. Yüksel N, Karabaş VL, Arslan A, Demirci A, Cağlar Y (2001) Ocular hemodynamics in pseudoexfoliation syndrome and pseudoexfoliation glaucoma. Ophthalmology 108(6): 1043-1049.

25. Martinez A, Sanchez M (2008) Retrobulber hemodynamic parameters in pseudoexfoliation syndrome and pseudoexfoliative glaucoma. Graefes Arch Clin Exp Ophthalmol 246(9): 1341-1349.

26. Ocakoglu O, Koyluoglu N, Kayiran A, Tamcelik N, Ozkan S (2004) Microvascular blood flow of the optic nerve head and peripapillary retina in unilateral exfoliation syndrome. Acta Ophthalmol Scand 82(1): 49-53.

27. Sommer A, Katz J, Quigley HA, Miller NR, Robin AL, et al. (1991) Clinically detectable nerve fiber atrophy precedes the onset of glaucomatous field loss. Arch Ophthalmol 109(1): 77-83.

28. Wollstein G, Schuman JS, Price LL, Aydin A, Stark PC, et al. (2005) Optical coherence tomography longitudinal evaluation of retinal nerve fiber layer thickness in glaucoma. Arch Ophthalmol 123(4): 464-470.

29. Curcio CA, Allen KA (1990) Topography of ganglion cells in human retina. J Comp Neurol 300(1): 1-25.

30. Seong M, Sung KR, Choi EH, Kang SY, Cho JW, et al. (2010) Macular and peripapillary retinal nerve fiber layer measurements by spectral domain optical coherence tomography in normal-tension glaucoma. Invest Ophthalmol Vis Sci 51(3): 1446-1452.

31. Kim NR, Lee ES, Seong GJ, Kim JH, An HG, et al. (2010) Structurefunction relationship and diagnostic value of macular ganglion cell complex measurement using Fourier-domain OCT in glaucoma. Invest Ophthalmol Vis Sci 51(9): 4646-4651.

32. Tan O, Li G, Lu AT, Varma R, Huang D, et al. (2008) Mapping of macular substructures with optical coherence tomography for glaucoma diagnosis. Ophthalmology 115(6): 949-956.

33. Mori S, Hangai M, Sakamoto A, Yoshimura N (2010) Spectral-domain optical coherence tomography measurement of macular volume for diagnosis in glaucoma. J Glaucoma 19(8): 528-534.

34. Na JH, Kook MS, Lee Y, Yu SJ, Choi J (2012) Detection of macular and circumpapillary structural loss in normal hemifield areas of glaucomatous eyes with localized visual field defects using spectraldomain optical coherence tomography. Graefes Arch Clin Exp Ophthalmol 250(4): 595-602.

35. Sevim MS, Buttanri B, Acar BT, Kahya A, Vural ET, et al. (2013) Ability of fourier-domain optical coherence tomography to detect retinal ganglion cell complex atrophy in glaucoma patients. J Glaucoma 22(7): 542-549.

36. Sorkhabi R, Rahbani MB, Ahoor MH, et al. Retinal Nerve Fiber Thickness in Patients with Exfoliation Syndrome. Iranian J Ophthalmol 24(2): 40-46.

37. Yüksel N, Altintaş O, Celik M, Ozkan B, Cağlar Y (2007) Analysis of retinal nerve fiber layer thickness in patients with pseudoexfoliation syndrome using optical coherence tomography. Ophthalmologica 221(5): 299-304. 
This work is licensed under Creative Commons Attribution 4.0 License DOI: $10.19080 /$ JOJO.2017.06.555677
Your next submission with Juniper Publishers will reach you the below assets

- Quality Editorial service

- Swift Peer Review

- Reprints availability

- E-prints Service

- Manuscript Podcast for convenient understanding

- Global attainment for your research

- Manuscript accessibility in different formats

( Pdf, E-pub, Full Text, Audio)

- Unceasing customer service

Track the below URL for one-step submission https://juniperpublishers.com/online-submission.php 